\title{
Development of the Simulation System of Multi-model H-beam Rolling
}

\author{
Kaiyue $\mathrm{Li}^{1,2, a}$, Qinhe Zhang ${ }^{1,2, \mathrm{~b}^{*}}$, and Yang Ren ${ }^{1,2, \mathrm{c}}$, Liming Wang ${ }^{1,2, \mathrm{~d}}$ \\ ${ }^{1}$ School of Mechanical Engineering, Shandong University, Jinan, China \\ ${ }^{2}$ Key Laboratory of High Efficiency and Clean Mechanical Manufacture of Ministry of Education, \\ Shandong University, Jinan 250061, China \\ a630140772@qq.com, b'zhangqh@sdu.edu.cn, c444320328@qq.com, 'w080810121@126.com
}

Keywords: H-beam, Re-meshing technology, Rolling process, Simulation system

Abstract. Simulation system plays an important role in the production process control and product optimization of multi-model H-beam hot rolling process. In this paper, an automatic modeling module was developed based on the customized application development method provided by ABAQUS, and the rolling process of HN900 $\times 300$ was taken as an example to conduct a simulation analysis. As a result, the automatic modeling, high pressure water descaling, cogging rolling, universal rolling and cooling were realized. Also, the surface temperature of H-beam was measured by thermal infrared imager at production site. It is found that the results of finite element simulation are basically consistent with the measuring results. The simulation system developed in this paper can improve the production process control of multi-model H-beam rolling.

\section{Introduction}

$\mathrm{H}$-beam is a high-performance section steel due to its advantage of optimized cross sectional area distribution and reasonable ratio of strength to weight. With the features of wide flange and thin web, H-beam has large section modulus, high bending resistance and excellent mechanical properties. Therefore, H-beam is widely used in plant construction, power generation facility, petrochemical industry, offshore drilling platform, subway engineering etc.

The rolling process of H-beam happens under high temperature and high pressure, involving the highly nonlinear plastic deformation process. Since the rolling information is hard to be obtained in real time as well the rolling process environment is comparatively complicated, it is difficult to control the quality of rolled workpiece. many scholars have conducted simulation analysis on the rolling process of section steel using large commercial finite element softwares. Phaniraj et al. [1] have used DEFORM to simulate the whole rolling process of $\mathrm{H}$-beam with thermo-mechanical coupling method, and obtain the distribution rule of the stress-strain field. He et al. [2, 3] has simulated and analyzed the metal flowing law and temperature field of medium-sized H-beam, in which the stress, strain and heat exchange have been considered. Zhang et al.[4] has applied the re-meshing technique to the simulation analysis process, and the validity is verified by the comparison of the measuring results and simulation results.

In this paper, a simulation system of multi-model H-beam rolling process was developed, which can realize the general simulation of rolling process of large, medium and small-sized H-beam, while avoiding the non-transparency of rolling information. It is helpful to improve the production process control and product optimization of multi-models of H-beam. 


\section{The theory and technology of finite element simulation}

Incremental step and mass scaling. The rolling process of $\mathrm{H}$-beam can be regarded as a quasi-static process as the rolling forming speed is relatively uniform and loading speed is relatively slow. In ABAQUS analysis, the equation of time increment can be given as

$$
\Delta t \leq\left(L_{\min } \sqrt{\frac{\rho}{\lambda+2 \mu}}, L_{\min }^{2} \cdot \frac{\rho \sigma}{2 k}\right)
$$

where $\Delta t$ is the time increment, $L_{\min }$ is the minimum element size, $\rho$ is the material density, $\lambda$

is the thermal conductivity, $\mu$ is the shear modulus, $\mathrm{c}$ is the velocity, $\mathrm{k}$ is the adiabatic exponent.

From Eq. (1), it can be found that the time increments are usually very small which would lead to some unnecessary calculations in the process of calculation of steel rolling. As a result, the calculation efficiency decreases. It can be seen from the Eq. (1) that , the time increment increases in proportion to mesh size and material density. Therefore, when the mesh density is kept constant, the calculation efficiency can be improved by increasing the material density to reduce the time increment. It can be seen from Eq. (1) that if density is magnified by 100 times, calculation speed will be increased by $10 \sim 100$ times. The simulation analysis process can be sped up by setting appropriate mass scale scaling value, based on the mass scaling technology provided by ABAQUS. In the rolling process of H-beam, it has been verified through several simulation practices that the analysis efficiency can be improved when the mass parameter is magnified 100 1000 times.

Finite element mesh technology. Lagrangian method is often be adopted in the meshing of traditional finite element model of continuous solid, in which the material model mesh attached to each cell, nodes and meshes are movable and deformable. However, when involving simulation with large deformation, mesh cell will become distorted and the deterioration of mesh quality will lead to the discontinue of simulation. Therefore, in the case of simulation with mesh distortion, traditional Lagrangian method cannot be used to complete the simulation of rolling process of $\mathrm{H}$-beam. Another common approach of meshing is Eulerian method. By Eulerian method, material has a high degree of freedom with the fixed mesh space being given to material and nodes being fixed, it can effectively solve the problems of large mesh distortion, material damage, fluids etc. But the Eulerian method is unable to be used to the complicated contact simulation analysis because of its low material boundary. In addition, ABAQUS also provides an ALE adaptive mesh technology [5]. This technology can realize adaptive mesh division by combining the advantages of both Lagrangian and Eulerian methods. also, nodes are allowed to move with the material distortion, thus the quality of meshes can be ensured by re-meshing. Therefore, the ALE adaptive mesh technology is adopted in this paper, as it can effectively solve the problems of mesh distortion and ensure the accuracy of simulation.

\section{The development of simulation system of rolling process}

The technology base of simulation system development. ABAQUS/CAE is an interactive graphical environment [6]which can easily generate or input analysis models and realize the model creation, job submission, assessment, test etc. A scripting interface is provided by ABAQUS, through which users can directly communicate with ABAQUS/CAE with bypassing GUI interface. 
With the help of Python programming language, the efficiency of pretreatment process can be increased by directly input the modeling parameters.

The overall design and development of simulation system. ABAQUS provides a customized application development method, and the startup interface can be directly modified by the user customized programs, namely lawful Python program, which can be activated when launching ABAQUS/CAE. It canbe realized the loading, modification and hiding of ABAQUS built-in modules as well as the adding of custom GUI modules. The principle and structure of a customized application are shown in Fig. 1. First of all, call the application through launching a script. The application is the highest level response for the simulation system as well as the interface of the system with Windows desktop. Then the main window of simulation system pops up, in which the structure of main interface including menus, toolbars and other elements can be defined. Specified modules and tool sets are loaded in the window, and different menus and buttons correspond to specific GUI response mechanism, by which the display of a dialog box can be controlled and the response of a button and an instruction can be assigned.

Custom tool set function contains the frame structure of "rolling menu", which includes 5 sub-menus: automatic modeling, high pressure water descaling, rough rolling, universal rolling and cooling on the cooling bed. Custom tool set function calls different GUI mechanisms through the keywords of AFXMenucommand and AFXMode. ID_ACTIVEATE, then the dialog box interfaces of corresponding sub-modules pop up for users to input parameters and conduct subsequent operations. Program, the main interface window, tool sets and the corresponding several GUI display modules constitute the overall structure of the system, which can achieve multi-step loading and startup by function call.

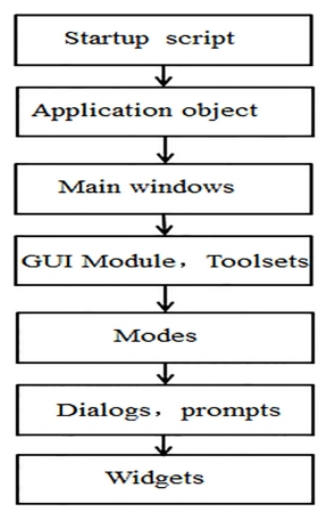

Fig.1 Customized application flowchart.

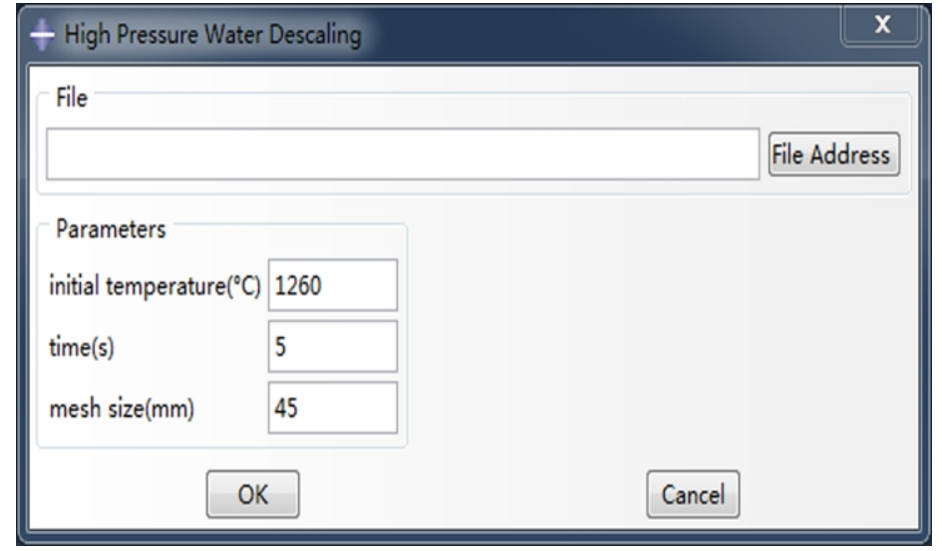

Fig. 2 High pressure water descaling.

\section{The design and development of simulation system sub-module}

High-pressure water descaling. High-pressure water descaling process consists of three main parameters: Initial rolling temperature, high pressure water descaling time, and track running time, which need to be manually input. In the script, the assignment of initial temperature is realized through the "predefined field" function, and the assignment of time length is achieved through the time setting of "analysis step". Due to relatively weak two-dimensional modeling capability, corresponding CAD models need to be introduced to ABAQUS. Therefore, it is necessary to develop an automatic modeling module to introduce the two-dimensional engineering drawings so as to generate the two-dimensional geometric model of rolled pieces. The dialog box interface of processing module before high-pressure water descaling is shown in Fig.2. 
Rough rolling or edge rolling. In the rolling process of H-beam, the ODB files of the model and information of rolled workpieces need to be introduced from the previous process so as to achieve continuous rolling. Roller draft can be generated by introducting the DXF file to improve the efficiency of modeling. The roller can be determined to be rigid roller or deformable roller according to the requirements. Rigid modeling can achieve rapid analysis and improve analysis efficiency while deformable modeling can analyze the deformation and stress of rollers. The dialog box interface of processing module before rough rolling or edge rolling is shown in Fig. 3.

Universal rolling. In the rolling process of H-beam, universal rolling stage is composed of two forms, UR and UF [7]. The UR and UF have the same space assembly mode while they are different in roller shapes. therefore, their pre-treatment operation and script setting basically have no difference, and the modeling and simulation can be accomplished just by calling corresponding rollers according to different situations. The dialog box of processing module before universal rolling is shown in Fig. 4.

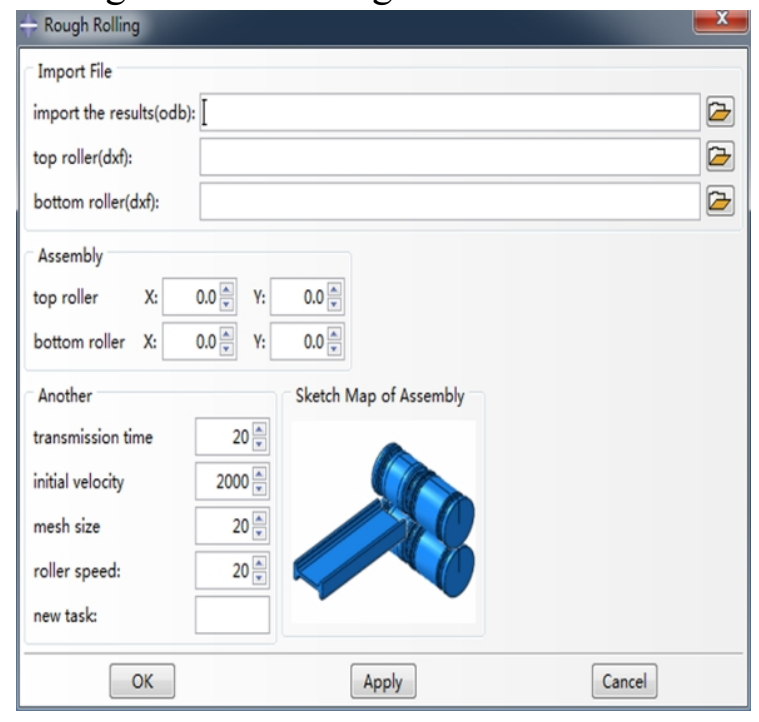

Fig.3 Rough rolling or edge rolling.

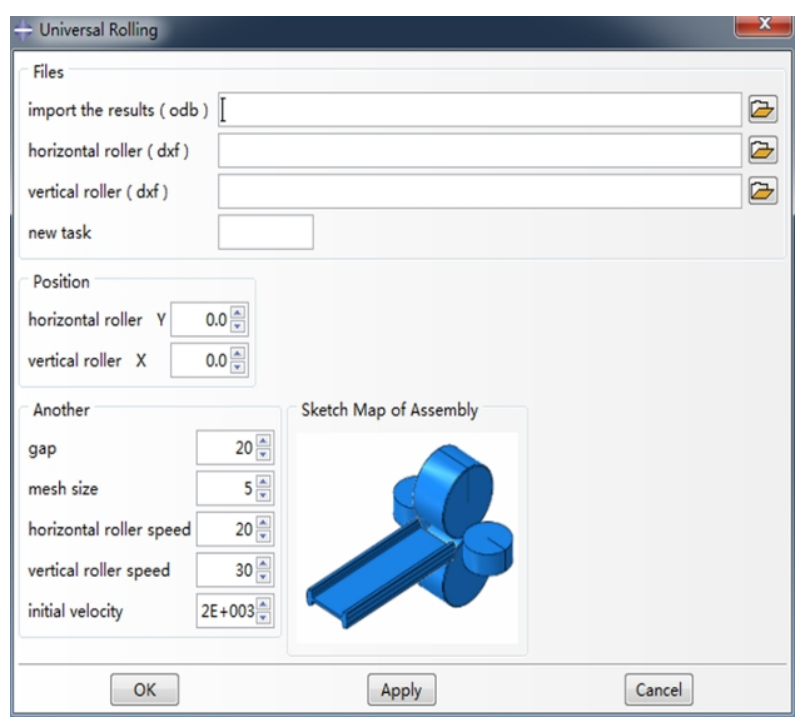

Fig.4 Universal rolling.

Cooling after rolling. The final stage of rolling of $\mathrm{H}$-beam is cooling, including three stages which are track running, laminar cooling and air cooling in cooling bed[7]. Water treatment process is based on the surface heat radiation energy density in field process, and the water cooling time is about 5 seconds. Air cooling process involves two kinds of cooling methods: heat radiation and heat convection. The convective heat transfer coefficient of rolled pieces in the air is $h=10 J \cdot s^{-1} m^{-2} K^{-1}$, and the air cooling time is about 45 minutes. The dialog box of cooling and processing module after rolling is shown in Fig.5.

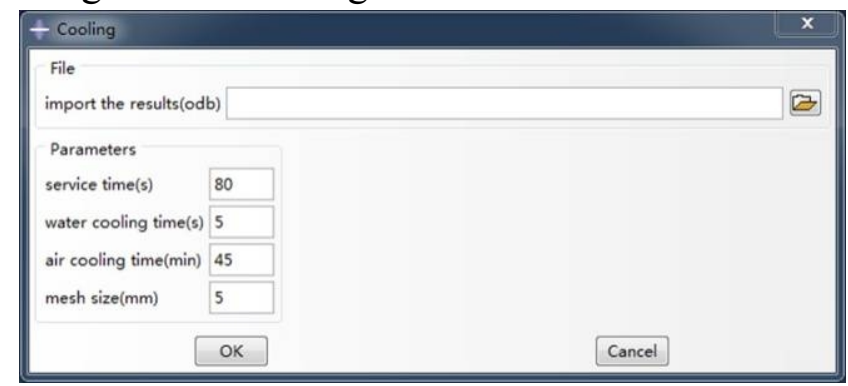

Fig.5 The dialog box of cooling and processing module after rolling. 


\section{Verification of simulation results}

The rolling process of HN900×300 section was taken as an example to make simulation analysis, and the surface temperature of $\mathrm{H}$-beam was measured using thermal infrared imager at production site. The comparison of simulation results with measuring results is shown in Fig.6 and Fig.7. It can be seen from Fig. 6 and Fig.7 that the results of finite element simulation are basically consistent with the measuring results, with an average relative error of $\pm 6 \%$ and an absolute error of $\pm 27^{\circ}$.

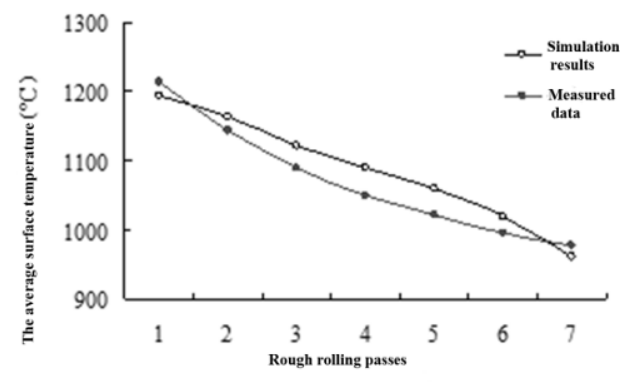

Fig.6 Rough rolling passes

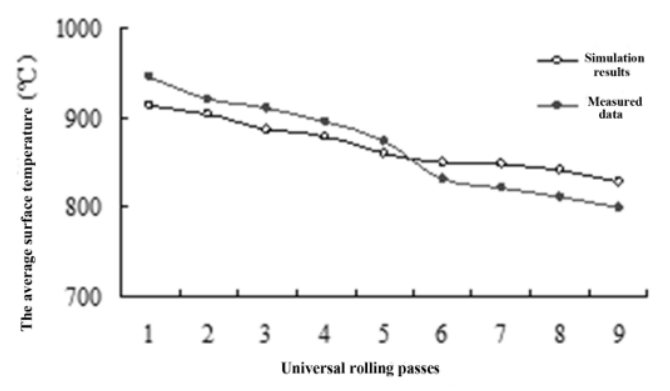

Fig.7 Universal rolling passes

\section{Summary}

A simulation system of multi-model H-beam rolling has been developed in this paper. In the rolling system, the information of original meshes is mapped to newly established meshes through certain algorithm by the re-meshing technology to ensure accurate and complete transfer of rolling information. The comparison of simulation results with measuring results is discussed, which proves the correctness of simulation. This method has the vital practical significance and the theory value in designing groove, optimizing rolling craft parameter and developing new H-beam, as it can provide value reference to the rolling practice.

\section{Acknowledgements}

The work is supported by grants from Science and Technology Development Program of Shandong (Grant No. 2010GGX10421) and Major National Science and Technology Program for "Top Grade CNC Machine Tools and Basic Manufacturing Equipment”, Grant No.: 2011ZX04016-091.

\section{References}

[1] M.P. Phaniraj, B.B. Behera, A.K. Lahiri. Thermo-mechanical modeling of two phase rolling and microstructure evolution in the hot strip mill Part I. Prediction of rolling loads and finish rolling temperature. J. Mater. Process. Tech. 170 (2005) 323-335.

[2] Q.Q. He, Q.H. Zhang, K.Q. Liu. Steel rolling finite element simulation. Journal of Wuhan University of Technology, 28 (2006) 118-120.

[3] Q.Q. He, Q.H. Zhang, K.Q. Liu, et al. Temperature-displacement simulation of shape-metal 9-pass-cogging process. (2006) 1136-1140.

[4] H.L. Zhang, Q. H. Zhang, Q.Q. He, et al. Simulation of shape metal in hot finishing rolling process. ICFDM, 4 (2008) 1180-1184.

[5] ALE adaptive meshing. ABAQUS Analysis User's Manual. Dassault Systèmes Simulia Corp., 2012. 
[6] J.H. Zhang, L Ding, Proficient in ABAQUS basic introduction and cases, Electronics industry Press, Beijing, 2012

[7] P.Q. Wang, Thermal Mechanical Coupled 3D Finite Element Simulation of Large H-Beam in Hot Rolling Process. Applied Mechanics and Materials. 281(2013) 484-489. 